\title{
Pseudo-Jahn-Teller effects in two-dimensional silicene, germanene and stanene: a crystal orbital vibronic coupling density analysis
}

\author{
MAITRAYEE GHOSH and AYAN DATTA* (D) \\ Department of Spectroscopy, Indian Association for the Cultivation of Science, Jadavpur 700032, India \\ *Author for correspondence (spad@iacs.res.in)
}

MS received 19 February 2018; accepted 21 May 2018; published online 22 August 2018

\begin{abstract}
The presence of the pseudo-Jahn-Teller (PJT) effect has been investigated in the heavier analogues of graphene, namely silicene, germanene and stanene, by applying the orbital vibronic coupling density theory. In order to do so, we have made a vis-a-vis analogy with their respective planar, honeycomb molecular cluster models, namely hexasilabenzene $\left(\mathrm{Si}_{6} \mathrm{H}_{6}\right)$, hexagermabenzene $\left(\mathrm{Ge}_{6} \mathrm{H}_{6}\right)$ and hexastannabenzene $\left(\mathrm{Sn}_{6} \mathrm{H}_{6}\right)$. One-to-one mapping of the occupied crystal orbitals and unoccupied crystal orbitals in two-dimensional (2D) Si, Ge and $\mathrm{Sn}$ systems to the occupied molecular orbitals and unoccupied molecular orbitals of the corresponding molecular units are used to identify PJT-active bands and compute the crystal orbital vibronic coupling density (c-OVCD) and crystal orbital vibronic coupling constants (c-OVCCs). c-OVCD and c-OVCC show the local picture of the PJT coupling in these 2D systems. This article exemplifies the fruitfulness of deciphering the structural aspects in materials based on orbitals of their corresponding simple molecular units-a reductionist quantum chemical approach to materials.
\end{abstract}

Keywords. Inorganic graphene analogues; vibronic effects; Jahn-Teller effects; symmetry breaking; orbitals.

\section{Introduction}

The phenomenal discovery of the first two-dimensional (2D) material, graphene, by the exfoliation of a monolayer of graphite using an adhesive tape by Novoselov et al [1] in 2004, which in itself is a milestone in the history of global science and technology, has subsequently led to the scientific interests and discovery of its $\mathrm{Si}, \mathrm{Ge}$ and $\mathrm{Sn}$ counterparts: silicene by epitaxial deposition on silver [2-5], iridium surface [6] and zirconium bromide [7], germanene synthesis by epitaxial deposition on gold surface [8,9] and stanene [10], respectively. The wide applications of graphene have renewed interests towards heavier analogues of Group IV 2D materials, viz., silicene, germanene and stanene. Silicene and germanene may have exotic applications, especially in the semiconductor industry, due to their tuneable band gap in electric field. All these emerging applications of Group IV layered materials can be ascribed to their molecular structural and electronic properties.

Previous theoretical calculations by our group [11-13] and Molina et al [14] and Soto et al [15,16] have shown that silicene, germanene and stanene have inherent out-of-plane distortions in their structures. The cluster models predict an average puckering angle of $33.7^{\circ}$ for $\mathrm{Si}_{6} \mathrm{H}_{6}$ at B3PW91/TZVP level of theory [13] and for $\mathrm{Ge}_{6} \mathrm{H}_{6}$, the buckling angle (torsional angle defined by any four adjacent Ge atoms) is $48.8^{\circ}$ at PBE0 level [11]. Soto et al [16] have concluded that the buckling parameter $\delta$ increases down Group IV, being the maximum of $0.92 \AA$ for Sn layers. This is attributed to spin-orbit coupling, which also increases in the same order [16]. Bersuker [17] has established that the deviations from planarity in electronically non-degenerate molecules occur solely due to the pseudo-Jahn-Teller effects (PJTEs). PJTE has been attributed to the distortions in several molecular systems $[11-16,18]$ and therefore can be a unifying concept to explain structural diversity in many molecules and materials [17,19-21].

For any pseudo-Jahn-Teller (PJT) active system, the linear vibronic coupling constant, or more commonly known as the vibronic coupling constant (VCC) (also called derivative coupling), is mathematically formulated as

$$
V_{\beta \gamma}=\left\langle\beta\left|\frac{\partial \hat{H}}{\partial Q_{\alpha}}\right| \gamma\right\rangle,
$$

where $\beta$ and $\gamma$ represent the two wavefunctions of the non-degenerate ground electronic state and an excited electronic state, respectively, $Q$ is the nuclear displacement coordinate along the frequency mode $\alpha$ and $\hat{H}$ is the adiabatic electronic Hamiltonian operator. For small distortions 
in $Q$ along $\alpha$ mode, the total force constant is given as

$$
K=K_{0}-\frac{V_{\beta \gamma}^{2}}{2 \Delta}
$$

$K$ becomes negative when the condition of instability holds true, that is, $K_{0}<V_{\beta \gamma}^{2} / 2 \Delta[17,19,22]$, where $\Delta$ is the occupied molecular orbital (OMO)-unoccupied molecular orbital (UMO) gap. When more than one excited state interacts with the ground state, the softening of the adiabatic force constant also includes contributions from the corresponding VCCs. Recently, we have shown that structural distortions in carbon sulphide $\left(\mathrm{C}_{6} \mathrm{~S}_{8}\right)$ and its suppression in S-oxygenated dithiine $\left(\mathrm{C}_{4} \mathrm{~S}_{4}\left(\mathrm{SO}_{2}\right)_{2}\right)$ are four-state and two-state PJT problems, respectively [23].

In the last decade, Sato and co-workers [24-28] have successfully developed the theory of vibronic coupling density (VCD) to study the vibronic coupling and consequently PJTE of several molecular systems like $\mathrm{C}_{3} \mathrm{H}_{3}$ [24], $\mathrm{NH}_{3}$ [24], anthracene [24], naphthalene anion [28], bi-benzene [29], cyclopentadienyl radical [30] and decasilanaphthalene [14] among others. VCD has also been successful at predicting the chemical reactivity of giant molecules like fullerenes, including $\mathrm{C}_{60}$ [2] and $\mathrm{C}_{70}$ [27,31]. Recently, the VCD theory was successfully applied to inelastic tunnelling spectroscopy [24,32] and designing carrier-transport molecules [33-35]. Before we use it for periodic systems, it is worthwhile to discuss the basic equations of the VCD theory.

The molecular Hamiltonian is defined as

$$
\mathbf{H}(\mathbf{r}, \mathbf{R})=\mathbf{H}_{\mathrm{e}}(\mathbf{r}, \mathbf{R})+\mathbf{T}_{\mathrm{n}}(\mathbf{R}),
$$

where $\mathbf{r}$ and $\mathbf{R}$ represent the electronic and nuclear coordinates, respectively. The vibronic coupling operator is defined as [36]

$$
\hat{V} Q_{\alpha}=\left(\frac{\partial \hat{H}}{\partial Q_{\alpha}}\right)_{R_{0}} Q_{\alpha}
$$

For the $n$th state, the on-diagonal VCC is defined as [24,36]

$$
\hat{V}_{n}^{\alpha}=\left\langle\Psi_{n}\left(R_{0}, r\right)\left|\hat{V}_{\alpha}\right| \Psi_{n}\left(R_{0}, r\right)\right\rangle+\left(\frac{\partial U_{n n}}{\partial Q_{\alpha}}\right)_{R_{0}} .
$$

Sato defines the on-diagonal VCD $\eta_{\alpha}$ as the product of the electron density difference $\Delta \rho$ of the ground state and an ionized state and the potential derivative $v_{\alpha}$ with respect to the mass-weighted normal coordinate $Q_{\alpha}[24,36,37]$ :

$$
\eta_{\alpha}=\Delta \rho(r) v_{\alpha}(r)
$$

Thus the on-diagonal VCC becomes

$$
V_{n}^{\alpha}=\int \eta_{\alpha}(r) d^{3} r=\int \Delta \rho(r) v_{\alpha}(r) d^{3} r
$$

Equations (5) and (7) are the same since they both represent on-diagonal VCC.

Here, the potential derivative $v_{\alpha}$ is the derivative of the potential $u(r)$ acting on a single electron from all nuclei. Thus, the potential derivative is mathematically formulated as [24, $36,37]$

$$
v_{\alpha}(r)=\left(\frac{\partial u(r)}{\partial Q_{\alpha}}\right)_{R_{0}} .
$$

The off-diagonal VCD for two different states $m$ and $n, \eta_{m n}^{\alpha}$, can be written as $[24,36]$

$$
\eta_{m n}^{\alpha}\left(r_{1}\right)=\rho_{m n}\left(r_{1}\right) v_{\alpha}\left(r_{1}\right)
$$

Here $\rho_{m n}\left(r_{1}\right)$ is the overlap density of the two states, $m$ and $n$, and $v_{\alpha}\left(r_{1}\right)$ is the potential derivative as described earlier. The off-diagonal VCC is defined as the spatial integration over the off-diagonal VCD:

$$
V_{m n}^{\alpha}=\int \eta_{m n}^{\alpha}\left(r_{1}\right) d^{3} r=\int \rho_{m n}\left(r_{1}\right) v_{\alpha}\left(r_{1}\right) d^{3} r_{1}
$$

VCD can be further broken down into an orbital level approach and this gives rise to an orbital vibronic coupling density (OVCD) [24]. In the present study, we replace the state-based analysis by a more local picture of the orbital analysis. Therefore, in the subsequent sections, we explore the orbital overlap densities, the potential derivative and the OVCDs and the related orbital vibronic coupling constants (OVCCs).

With this background knowledge, we aim to examine the possibility of expanding the OVCD theory from molecular systems to periodic systems (henceforth called c-OVCD) and hence to study the PJT-induced distortions in silicene, germanene and stanene from c-OVCD point of view. In this regard, we have presented c-OVCD analyses with one-to-one comparison of the crystal orbitals (COs) of the honeycomb lattices of each periodic system to molecular orbitals (MOs) of hexagonal molecular analogues: silicene to $\mathrm{Si}_{6} \mathrm{H}_{6}$, germanene to $\mathrm{Ge}_{6} \mathrm{H}_{6}$ and stanene to $\mathrm{Sn}_{6} \mathrm{H}_{6}$. The off-diagonal OVCDs/c-OVCDs and OVCCs/crystal orbital vibronic coupling constants (c-OVCCs) have been computed for every periodic and molecular system in the context of PJTE-induced symmetry breaking.

\section{Computational details}

All the calculations have been performed using the Gaussian 09 suite of programs [38] and Gauss View 05 and VESTA have been used for visualization of $2 \times 2$ hexagonal lattices, MOs, COs, the orbital overlap densities, potential derivatives and OVCDs (c-OVCDs). The PBEPBE functional was used for structure optimizations and frequency calculations. For the silicene- $-\mathrm{Si}_{6} \mathrm{H}_{6}$ and germanene- $-\mathrm{Ge}_{6} \mathrm{H}_{6}$ 

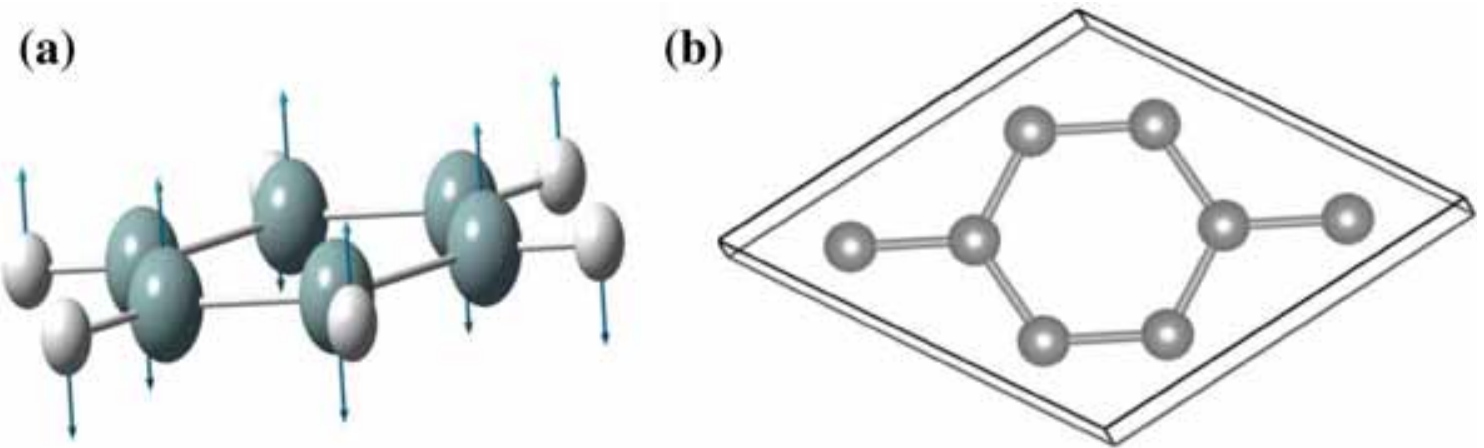

Figure 1. (a) Optimized structure of $\mathrm{X}_{6} \mathrm{H}_{6}(\mathrm{X}=\mathrm{Si}, \mathrm{Ge}, \mathrm{Sn})$ at $\mathrm{D}_{6 \mathrm{~h}}$ symmetry containing vibrational instability along the $b_{2 g}$ mode. The arrows show the displacement vectors along this mode. Colour code: dark grey atoms represent $\mathrm{X}(\mathrm{X}=\mathrm{Si}, \mathrm{Ge}, \mathrm{Sn})$ and the light grey atoms are hydrogens. (b) Structure of the planar hexagonal $2 \times 2$ unit cell of heavier graphene analogues: silicene $(a=3.93 \AA, b=3.92 \AA)$, germanene $(a=4.10 \AA, b=4.11 \AA)$ and stanene $(a=4.83 \AA$, $b=4.83 \AA)$. Note that these structures have buckling instability.

pairs, a TZVP basis set was utilized. In the case of the stanene $-\mathrm{Sn}_{6} \mathrm{H}_{6}$ pair, the LANL2DZ basis set was used. For the molecular units, namely $\mathrm{Si}_{6} \mathrm{H}_{6}, \mathrm{Ge}_{6} \mathrm{H}_{6}$ and $\mathrm{Sn}_{6} \mathrm{H}_{6}$, the $\mathrm{D}_{6} \mathrm{~h}$ point group was used for all calculations. Frequencies for layered planar materials $(2 \times 2$ unit cells, see figure $1 b)$ have been obtained numerically using the relation freq=numer keyword available in Gaussian 09 using periodic boundary conditions (PBCs) because these layered materials do not have a definite point group unlike their molecular analogues. The instability of the planar periodic layers has been additionally verified by relaxation and phonon calculations for silicene, germanene and stanene using the Vienna $\mathrm{Ab}$ Initio Simulation Package (VASP) [39,40] (see the supplementary information).

After optimization and frequency calculations, the normal mode corresponding to the $\mathrm{D}_{3 \mathrm{~d}}$ instability $\left(\mathrm{b}_{2 \mathrm{~g}}\right.$ mode) was identified. However, for the periodic systems, since numerical frequencies are computed the symmetry of the mode of instability is not determined. To choose the correct frequency mode, we have animated the normal modes in silicene, germanene and stanene and found out the vibrational mode that is responsible for the displacement of the planar $\mathrm{D}_{6 \mathrm{~h}}$ structure to chair-like $\mathrm{D}_{3 \mathrm{~d}}$ conformation (buckling). Visual inspection of this mode is rather straightforward as the $\mathrm{b}_{2 \mathrm{~g}}$ mode displaces the $z$-axis coordinates only, leaving the $x$ - and $y$-axes unchanged. This was done keeping in mind our aim for comparison with the hexagonal, molecular ring units, that is, $\mathrm{Si}_{6} \mathrm{H}_{6}, \mathrm{Ge}_{6} \mathrm{H}_{6}$, and $\mathrm{Sn}_{6} \mathrm{H}_{6}$. Hence, to keep the periodic systems and their molecular cousins at par, the vibrational frequency mode that is similar to the $\mathrm{b}_{2 \mathrm{~g}}$ mode in $\mathrm{Si}_{6} \mathrm{H}_{6}$, $\mathrm{Ge}_{6} \mathrm{H}_{6}$, and $\mathrm{Sn}_{6} \mathrm{H}_{6}$ that transforms planar $\mathrm{D}_{6 \mathrm{~h}}$ conformation to off-the-plane, chair-like $\mathrm{D}_{3 \mathrm{~d}}$ conformation became the best fit for our study.

All the planar structures (molecules and their periodic analogues) were displaced by 0.01 a.u. along the $b_{2 g}$ modes, subsequent to which single-point energy calculations were performed followed by the generation of the corresponding formatted check-point files. They were used to compute the potential derivative, $v_{\alpha}$ (through numerical forward difference between the undistorted potential $\left(r_{b_{2 \mathrm{~g}}}=0.00\right.$ a.u.) and potential at $r_{b_{2 \mathrm{~g}}}=0.01$ a.u.). The formatted check-point files of both the undistorted and distorted structures are used to create the cube files of the MOs and COs, using the cubegen utility in Gaussian 09 [38]. We have used the default criterion for the number of points per side of the cube. This results in $80 \times 80 \times 80$ points evenly distributed over a rectangular grid generated automatically by the program [38]. Further, multiplication, scaling and subtraction of cube files to generate the overlap densities and electron density differences of the MOs, potential derivative and the vibronic coupling densities have been performed using the cubman utility available in Gaussian 09 . The scalar product of the overlap density of any two specific MOs (COs) and the potential derivative is the off-diagonal OVCD (c-OVCD) between those two orbitals. Finally, we have calculated the overall off-diagonal OVCCs by summing up the absolute values for the individual offdiagonal OVCCs at each grid point through in-house Fortran 90 codes.

\section{Results and discussion}

Analytical frequency calculations on planar $\mathrm{Si}_{6} \mathrm{H}_{6}, \mathrm{Ge}_{6} \mathrm{H}_{6}$ and $\mathrm{Sn}_{6} \mathrm{H}_{6}$ with imaginary frequencies at $\mathrm{D}_{6 \mathrm{~h}}$ point group symmetry reveal that the $b_{2 g}$ mode accounts for the $D_{6 h}$ to $\mathrm{D}_{3 \mathrm{~d}}$ symmetry breaking (figure 1a). Based on the character table and product table at $\mathrm{D}_{6 \mathrm{~h}}$ point group, the following combinations for the occupied molecular orbitals (OMOs) and unoccupied molecular orbitals (UMOs), namely $\mathrm{a}_{1 \mathrm{~g}} \otimes \mathrm{b}_{2 \mathrm{~g}}$, $a_{2 g} \otimes b_{1 g}, e_{1 g} \otimes e_{2 g}, a_{1 u} \otimes b_{2 u}, a_{2 u} \otimes b_{1 u}$ and $e_{1 u} \otimes e_{2 u}$, can undergo vibronic mixing along the $b_{2 g}$ mode to reduce its symmetry from $\mathrm{D}_{6 \mathrm{~h}}$.

Irrespective of the magnitude of the energy differences (large or small) between the PJT active OMO-UMO/occupied 


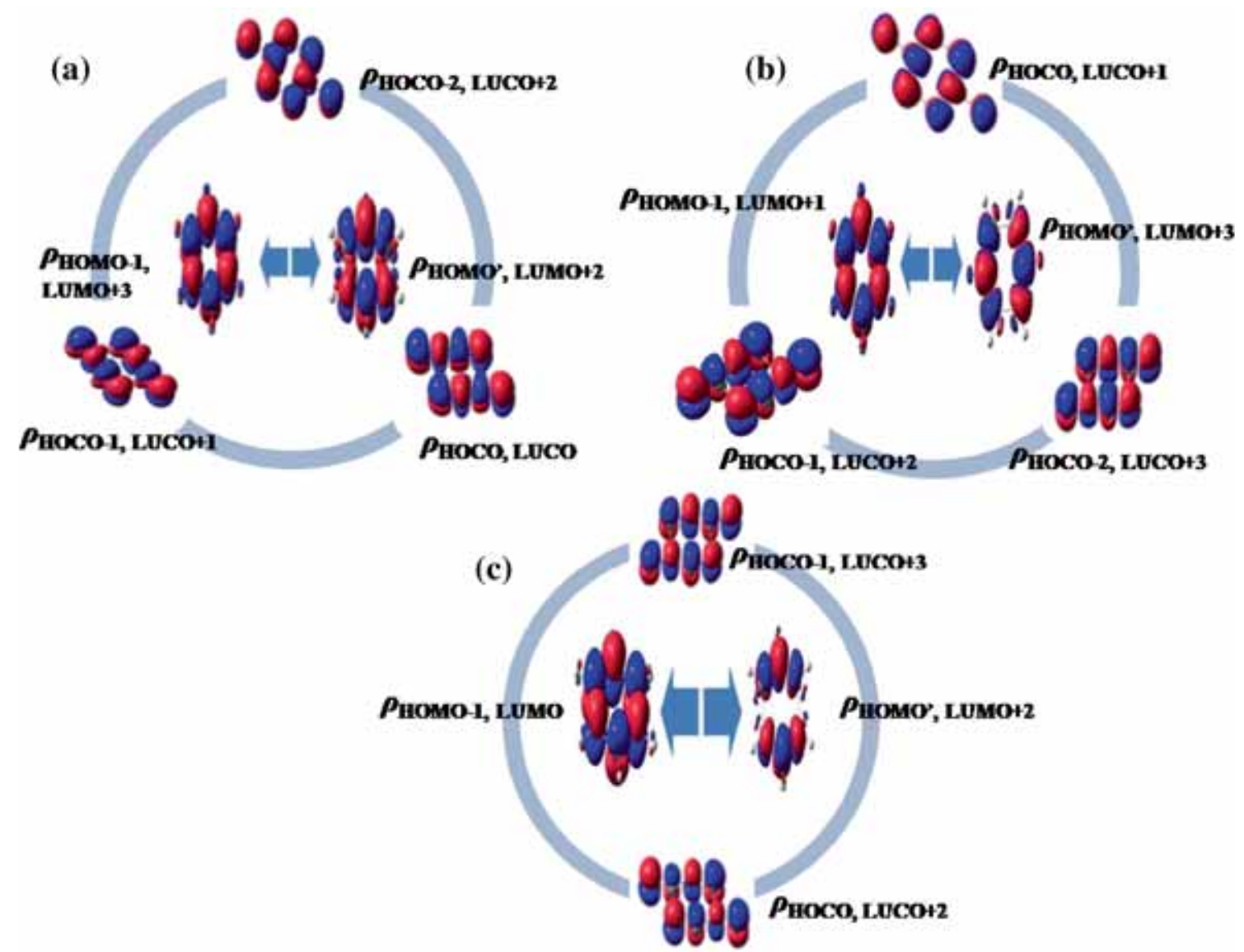

Figure 2. One-to-one mapping of the OMO-UMO and OCO-UCO overlap densities $(\rho)$ based on shapes and profile of the nodes for (a) hexasilabenzene $\left(\mathrm{Si}_{6} \mathrm{H}_{6}\right)$ and silicene, $(\mathbf{b})$ hexagermabenzene $\left(\mathrm{Ge}_{6} \mathrm{H}_{6}\right)$ and germanene and (c) hexastannabenzene $\left(\mathrm{Sn}_{6} \mathrm{H}_{6}\right)$ and stanene. [Note that the primed (') OMO/UMO indicates the particular orbital(s) that specifically undergoes vibronic coupling among the degenerate manifolds.]

crystal orbital (OCO)-unoccupied crystal orbital (UCO) pairs, no orbital combination can be eliminated solely based on high energy gap. As already discussed in equation (2), the PJT instability condition is affected by three key factors: the energy gap, the VCC and the primary force constant of the ground state $[14,19]$. Surely, the probability that the effect of the energy gap may be overruled by the strength of the other two factors cannot be ruled out. Therefore, a one-to-one mapping of the orbital overlap densities for the off-diagonal OVCDs for each of the three cases: (A) hexasilabenzene $\left(\mathrm{Si}_{6} \mathrm{H}_{6}\right)$ vs. silicene, (B) hexagermabenzene $\left(\mathrm{Ge}_{6} \mathrm{H}_{6}\right)$ vs. germanene and $(\mathrm{C})$ hexastannabenzene $\left(\mathrm{Sn}_{6} \mathrm{H}_{6}\right)$ vs. stanene, is essential for all the PJT-active OMO$\mathrm{UMO} / \mathrm{OCO}-\mathrm{UCO}$ combinations. It is to be noted that even if the $\mathrm{OMO}(\mathrm{UMO}) / \mathrm{OCO}(\mathrm{UCO})$ are degenerate; they have been treated individually due to their difference in wavefunctions.

From our frequency calculations in Gaussian 09, we report $v_{\mathrm{b}_{2 \mathrm{~g}}}=137.5 i, 141.2 i$ and $155.3 i \mathrm{~cm}^{-1}$ for $\mathrm{Si}_{6} \mathrm{H}_{6}$ (at PBEPBE/TZVP level), $\mathrm{Ge}_{6} \mathrm{H}_{6}$ (at PBEPBE/TZVP level) and $\mathrm{Sn}_{6} \mathrm{H}_{6}$ (at PBEPBE/LANL2DZ level), respectively. For silicene, germanene and stanene, the imaginary mode having the characteristic of a $b_{2 g}$ mode are 261.5i, 125.5i and $96.7 i \mathrm{~cm}^{-1}$ at the same level of theory as their molecular counterparts albeit using PBCs. Relevant phonon calculations [41] in VASP for the layered materials that corroborate the buckling distortion modes from the PBC calculations in G09 are included in the supplementary information.

As shown in figure 2, there are direct one-to-one mappings of the OMO-UMO and OCO-UCO overlap densities of the molecular units with their corresponding $2 \mathrm{D}$ analogues. For silicene, the HOCO-LUCO, HOCO-1-LUCO+1 and $\mathrm{HOCO}-2-\mathrm{LUCO}+2$ are correlated with the HOMO$1\left(\mathrm{a}_{2 \mathrm{u}}\right)-\mathrm{LUMO}+3\left(\mathrm{~b}_{1 \mathrm{u}}\right)$ and HOMO' $\left(\mathrm{e}_{1 \mathrm{~g}}\right)-\mathrm{LUMO}+2\left(\mathrm{e}_{2 \mathrm{~g}}\right)$ combination of the OMO-UMO for $\mathrm{Si}_{6} \mathrm{H}_{6}$. Similarly, for germanene, HOCO-LUCO-1, HOCO-1-LUCO+2 and HOCO2-LUCO+3 are correlated with HOMO-1 $\left(\mathrm{a}_{2 \mathrm{u}}\right)-\mathrm{LUMO}+$ $1\left(\mathrm{~b}_{1 \mathrm{u}}\right)$ and HOMO' $\left(\mathrm{e}_{1 \mathrm{~g}}\right)-\mathrm{LUMO}+3\left(\mathrm{e}_{2 \mathrm{~g}}\right)$ of $\mathrm{Ge}_{6} \mathrm{H}_{6}$ while for stanene, $\mathrm{HOCO}-1-\mathrm{LUCO}+3$ and $\mathrm{HOCO}-\mathrm{LUCO}+2$ are matched up with HOMO' $\left(\mathrm{e}_{1 \mathrm{~g}}\right)-\mathrm{LUMO}+2 \quad\left(\mathrm{e}_{2 \mathrm{~g}}\right)$ and HOMO-1 $\left(a_{2 u}\right)-L U M O\left(b_{1 u}\right)$ of $\operatorname{Sn}_{6} \mathrm{H}_{6}$. It is worthwhile to note that though there are several other combinations of the OMO-UMO in $\mathrm{Si}_{6} \mathrm{H}_{6}$ (see table 1), $\mathrm{Ge}_{6} \mathrm{H}_{6}$ (see table 2) 


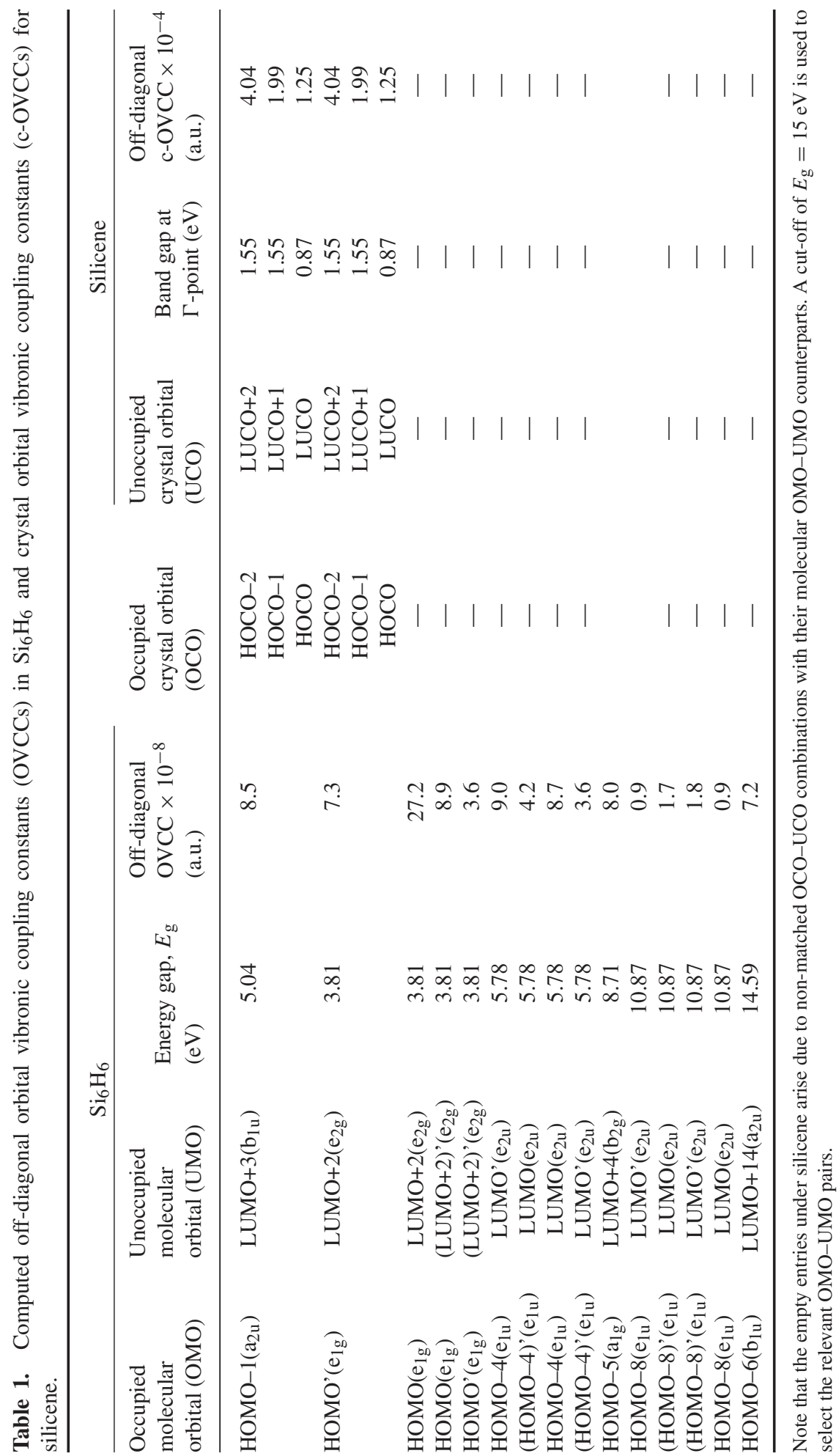




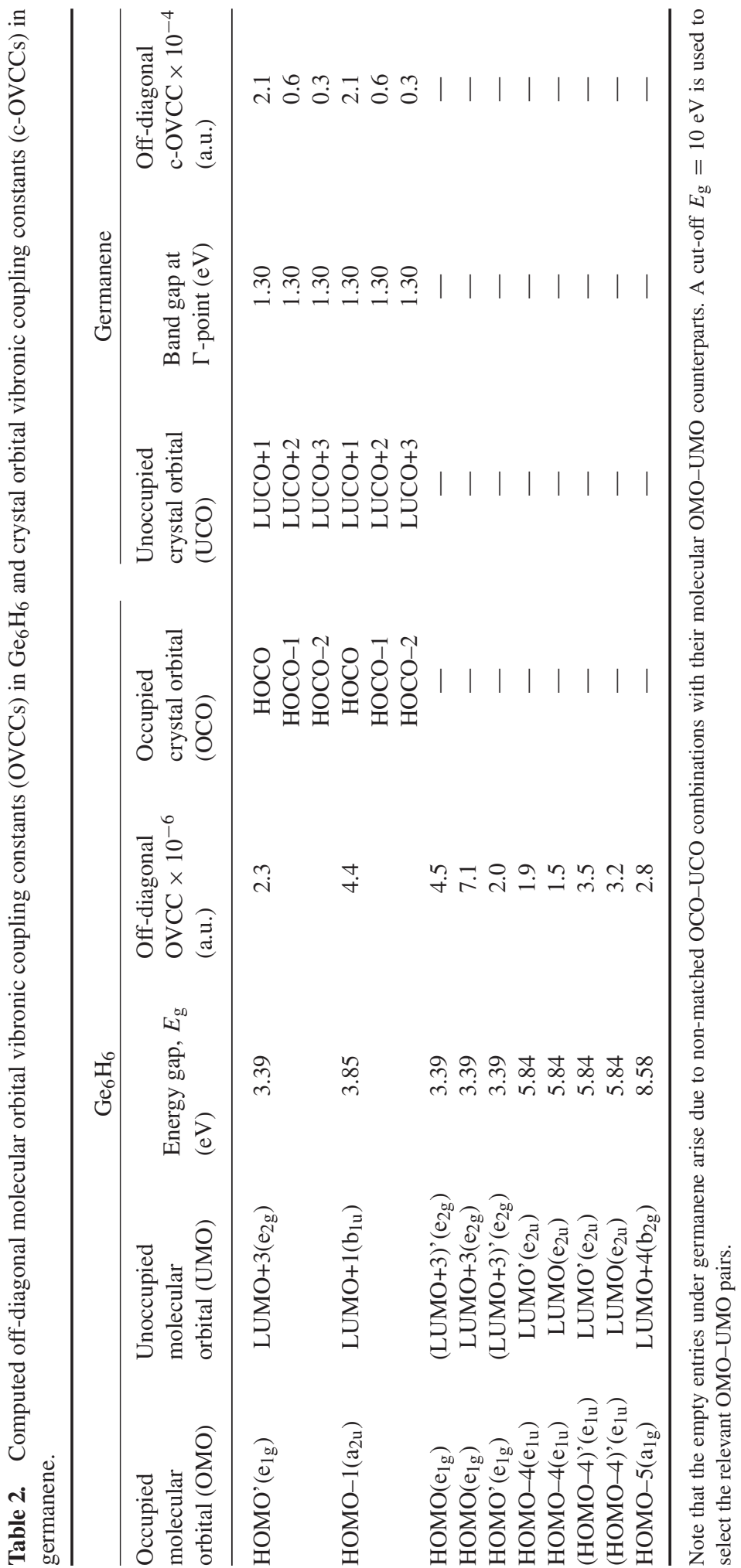




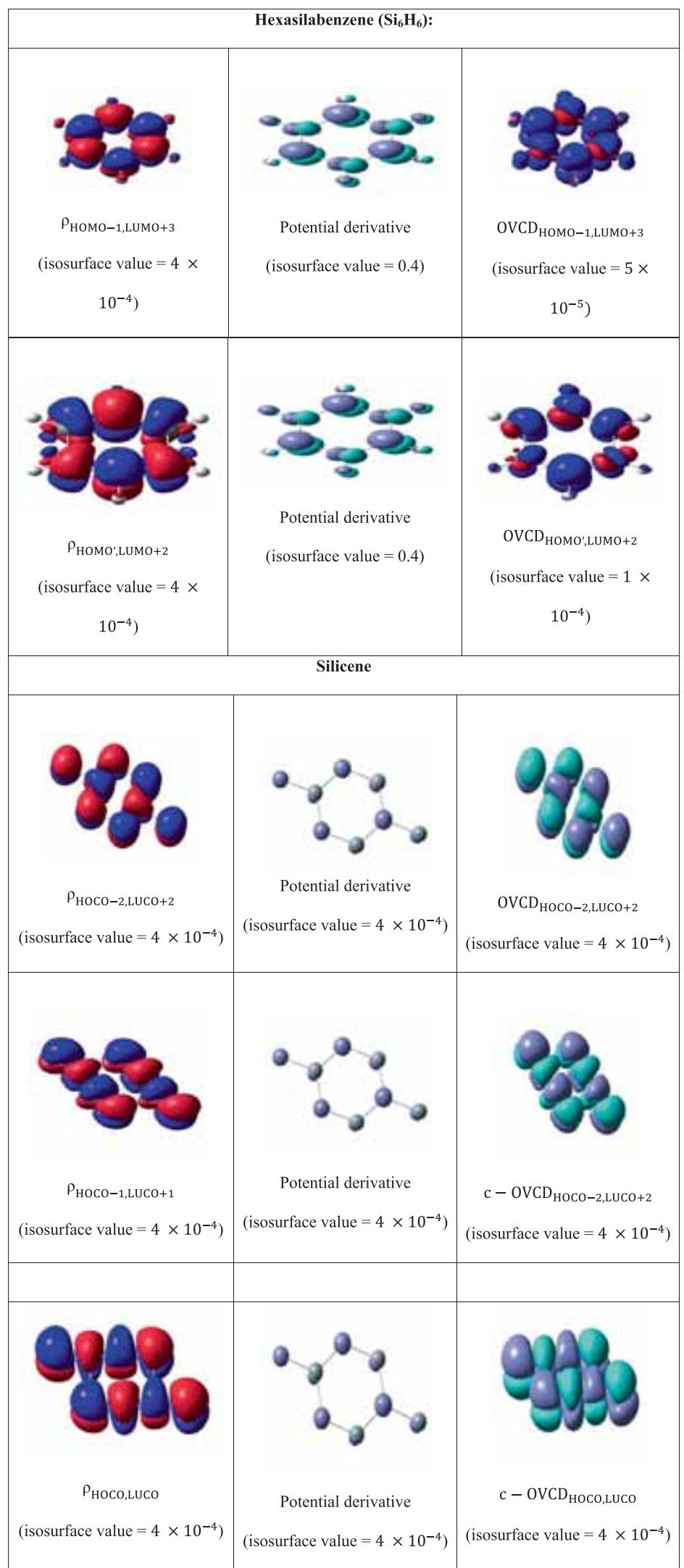

Figure 3. Off-diagonal OVCD and c-OVCD analyses for $\mathrm{Si}_{6} \mathrm{H}_{6}$ and silicene, respectively, based on the orbitals that are matched as shown in figure 2. Isosurfaces are reported in atomic units. 
and $\mathrm{Sn}_{6} \mathrm{H}_{6}$ (see supplementary information) that have the requisite symmetry combinations to undergo vibronic mixing along the $b_{2 g}$ mode, yet none of them are matched to relevant OCO-UCO pairs. Therefore, even though these OMO-UMO pairs contribute towards the OVCDs and OVCCs in their molecular analogues, they are not relevant in silicene, germanene and stanene.

Once the overlap densities $(\rho)$ between the PJT-active OMO-UMO/OCO-UCO are computed, their scalar products with the potential derivatives produce the relevant off-diagonal OVCDs/c-OVCDs. They are shown in figure 3 for $\mathrm{Si}_{6} \mathrm{H}_{6}$ and silicene and the same for $\mathrm{Ge}_{6} \mathrm{H}_{6}$ /germanene and $\mathrm{Sn}_{6} \mathrm{H}_{6} /$ stanene are shown in the supplementary information. Interestingly, the OVCDs of the molecular units and the c-OVCDs of their periodic counterparts have similar spatial profiles and hence, even at qualitative level, one can appreciate their direct mapping and correlation. As seen from table 1, the off-diagonal OVCCs in $\mathrm{Si}_{6} \mathrm{H}_{6}$ are $\sim \mathrm{O}\left(10^{-8}\right.$ a.u. $)$ with the largest contribution arising from the $\operatorname{HOMO}\left(\mathrm{e}_{1 \mathrm{~g}}\right)_{-}$ LUMO $+2\left(\mathrm{e}_{2 \mathrm{~g}}\right)$ pair having an energy gap $E_{\mathrm{g}}=3.81 \mathrm{eV}$. Note that other orbital combinations having the same energy gap have relatively smaller off-diagonal OVCCs. This justifies why we have treated degenerate orbitals separately. For silicene, only three OCO-UCO pairs, namely HOCO-LUCO, $\mathrm{HOCO}-1-\mathrm{LUCO}+1$ and $\mathrm{HOCO}-2-\mathrm{LUCO}+2$ having $E_{\mathrm{g}}$ (at $k=0, \Gamma$-point $)=0.87,1.55$ and $1.55 \mathrm{eV}$, respectively, are relevant. The c-OVCCs for silicene are $\sim \mathrm{O}\left(10^{-4}\right.$ a.u. $)$, the largest one arising from the HOCO-2-LUCO+2 pair, which again is not correlated with the smallest band gap at the $\Gamma$-point.

In the case of $\mathrm{Ge}_{6} \mathrm{H}_{6}$, the off-diagonal OVCCs are $\sim \mathrm{O}\left(10^{-6}\right.$ a.u. $)$ and of the several possible combinations for OMO-UMO (see table 2), the HOMO $\left(e_{1 g}\right)-L U M O+3\left(e_{2 g}\right)$ pair with $E_{\mathrm{g}}=3.39 \mathrm{eV}$ has the largest value of $7.1 \times$ $10^{-6}$ a.u. For germanene, the three possible combinations, $\mathrm{HOCO}-\mathrm{LOCO}+1\left(E_{\mathrm{g}}(\Gamma\right.$-point $\left.)=1.30 \mathrm{eV}\right), \mathrm{HOCO}-1-\mathrm{LU}$ $\mathrm{CO}+2\left(E_{\mathrm{g}}(\Gamma\right.$-point $\left.)=1.30 \mathrm{eV}\right)$ and HOCO-2-LUCO+3 $\left(E_{\mathrm{g}}(\Gamma\right.$-point $\left.)=1.30 \mathrm{eV}\right)$, lead to off-diagonal c-OVCCs of $2.0 \times 10^{-4}, 5.5 \times 10^{-5}$ and $3.3 \times 10^{-5}$ a.u., respectively. For $\mathrm{Sn}_{6} \mathrm{H}_{6}$, again more than one OMO-UMO contribute towards the off-diagonal OVCC $\left(\sim \mathrm{O}\left(10^{-6}\right.\right.$ a.u. $)$ and hence PJT distortion, the largest contribution arises from the $\operatorname{HOMO}\left(\mathrm{e}_{1 \mathrm{~g}}\right)-\mathrm{LUMO}+2\left(\mathrm{e}_{2 \mathrm{~g}}\right)$ pair with $E_{\mathrm{g}}=2.56 \mathrm{eV}$ and off-diagonal OVCC $=15.5 \times 10^{-6}$ a.u. For stanene, two OCO-UCO combinations that match those in $\mathrm{Sn}_{6} \mathrm{H}_{6}$ for the genesis of PJT, HOCO-LOCO+2 $\left(E_{\mathrm{g}}(\Gamma\right.$-point $\left.)=0.87 \mathrm{eV}\right)$ and $\mathrm{HOCO}-1-\mathrm{LUCO}+3\left(E_{\mathrm{g}}(\Gamma\right.$-point $\left.)=0.88 \mathrm{eV}\right)$, produce off-diagonal c-OVCCs of $9.7 \times 10^{-8}$ and $2.0 \times 10^{-8}$ a.u., respectively (see the supplementary information of OVCCs for $\mathrm{Sn}_{6} \mathrm{H}_{6}$ and stanene).

\section{Conclusions}

In this study, we have extended the concept of OVCD and OVCC to periodic systems. Based on a one-to-one mapping of the MOs of the small molecular analogues with the COs of periodic systems of large periodic systems, one can compute the relevant c-OVCD and c-OVCC. This approach provides a simple and intuitive method to determine local picture of the origin of symmetry breaking in materials based on vibronic interactions between the OCOs (UCOs). The utilization of symmetry (by analogy with their molecular/cluster models) and nodal characteristics of the COs is demonstrated in the test cases of PJT distortions for the silicene- $-\mathrm{Si}_{6} \mathrm{H}_{6}$, germanene$\mathrm{Ge}_{6} \mathrm{H}_{6}$ and stanene- $\mathrm{Sn}_{6} \mathrm{H}_{6}$ pairs. For the molecules as well as 2D layers, several combinations of the OMO-UMO/OCO$\mathrm{UCO}$ pairs of similar magnitudes for OVCC/c-OVCC exist. Hence, all of them contribute towards their PJT distortions. Clearly, selection of the most relevant vibronically active OMO-UMO/OCO-UCO pair purely based on their energy gap/band gap (at $\Gamma$-point) seems to be unjustified. A more logical option would be to select and compute the OVCC/cOVCC for all pairs that have similar magnitudes. One must note that determination of the relevant OCOs and UCOs for 2D materials by analogy with respect to planar molecular units is greatly assisted by the qualitatively different symmetries of the $\sigma$ and $\pi$ electrons. Also, our approach is essentially a simplification of the multimode problem as the vibronic coupling between electronic bands is also a band. Here, we sample the vibronic coupling at the $\Gamma$-point only to estimate the nature of instability qualitatively. For symmetry breaking in $3 \mathrm{D}$ materials like those involving octahedral to tetragonal phase-transitions, such one-to-one mapping is expected to be more complex. Nevertheless, in-principle one can correlate the COs in periodic systems to the MOs in smaller cluster models and use similar analogies to compute the c-OVCCs.

\section{SUPPLEMENTARY INFORMATION}

Cartesian coordinates of all molecules and periodic systems, phonon spectra of planar periodic systems with instability, OVCD and c-OVCD analyses of $\mathrm{Ge}_{6} \mathrm{H}_{6}$ - germanene and $\mathrm{Sn}_{6} \mathrm{H}_{6}$ - stanene, complete table for the OVCC and c-OVCC of $\mathrm{Sn}_{6} \mathrm{H}_{6}$ and stanene, complete Gaussian 09 reference.

\section{Acknowledgement}

This work was supported by the DST-SERB and BRNS.

\section{References}

[1] Novoselov K S, Giem A K, Morozov S V, Jiang D, Zhang Y, Dubonos S V et al 2004 Science 306666

[2] Lin C, Arafune R, Kawahara K, Kanno M, Tsukahara N, Minamitani E et al 2013 Phys. Rev. Lett. 110076801

[3] Feng B, Ding Z, Meng S, Yao Y, He X, Cheng P et al 2012 Nano Lett. 123507

[4] Chen L, Liu C C, Feng B, He X, Cheng P, Ding Z et al 2012 Phys. Rev. Lett. 109056804 
[5] Vogt P, Padova P D, Quaresima C, Avila J, Frantzekakis E, Asensio M C et al 2012 Phys. Rev. Lett. 108155501

[6] Meng L, Wang Y, Zhang L, Du S, Wu R, Li L et al 2013 Nano Lett. 13685

[7] Fleurence A, Friedlein R, Ozaki T, Kawai H, Wang Y and Yamada-Takamura Y 2012 Phys. Rev. Lett. 108245501

[8] Bianco E, Butler S, Jiang S, Restrepo O D, Windl W, Goldberger J E 2013 ACS Nano 74414

[9] Dávilla M E and Lay G L 2016 Sci. Rep. 620714

[10] Saxena S, Chaudhary R P and Shukla S 2016 Nat. Sci. Rep. 6 31073

[11] Nijamudheen A, Bhattacharjee R, Choudhury S and Datta A 2015 J. Phys. Chem. C 1193802

[12] Jose D and Datta A 2012 J. Phys. Chem. C 11624639

[13] Jose D and Datta A 2014 Acc. Chem. Res. 47593

[14] Molina B, Soto J R and Castro J J 2015 Chem. Phys. 460 97

[15] Soto J R, Molina B and Castro J J 2015 Phys. Chem. Chem. Phys. 177624

[16] Soto J R, Molina B and Castro J J 2017 MRS Adv. 21563

[17] Bersuker I B 2017 J. Phys.: Conf. Ser. 833012001

[18] Zhao J, Liu H, Yu Z, Quhe R, Zhou S, Wang Y et al 2016 Prog. Mater. Sci. 8324

[19] Bersuker I B 2013 Chem. Rev. 1131351

[20] Liu Y, Wang Y and Bersuker I B 2016 Sci. Rep. 623315

[21] Gorinchoy N N and Bersuker I B 2017 J. Phys.: Conf. Ser. 833 012010

[22] Kouchakzadeh G and Nori-Shargh D 2015 Phys. Chem. Chem. Phys. 1729251

[23] Pratik S M, Chowdhury C, Bhattacharjee R, Jahiruddin S and Datta A 2015 Chem. Phys. 460101
[24] Sato T, Uejima M, Iwahara N, Haruta N, Shizu K and Tanaka K 2013 J. Phys.: Conf. Ser. 428012010

[25] Tokunaga K, Sato T and Tanaka K 2007 J. Mol. Struct. 838 116

[26] Uejima M, Sato T, Tanaka K and Kaji H 2013 Phys. Chem. Chem. Phys. 1514006

[27] Haruta N, Sato T, Iwahara N and Tanaka K 2013 J. Phys.: Conf. Ser. 428012003

[28] Sato T, Tokunaga K and Tanaka K 2008 J. Phys. Chem. A 112 758

[29] Krasnenko V, Boltrushko V and Hizhnyakov V 2017 in: IOP Conference Series. J. Phys.: Conf. Ser. 833012009

[30] Sato T, Tokunaga K and Tanaka K 2006 J. Chem. Phys. 124 024314

[31] Haruta N, Sato T and Tanaka K 2012 J. Org. Chem. 779702

[32] Shizu K, Sato T and Tanaka K 2010 Nanoscale 22186

[33] Iwahara N, Sato N and Tanaka K 2012 J. Chem. Phys. 136 174315

[34] Shizu K, Sato T, Ito A, Tanaka K and Kaji H 2011 J. Mater. Chem. 216375

[35] Sato T, Shizu K, Tanaka K and Kaji H 2012 J. Photon. Energy 2021201

[36] Sato T 2017 J. Phys.: Conf. Ser. 833012020

[37] Sato T, Iwahara N, Haruta N and Tanaka K 2012 Chem. Phys. Lett. $\mathbf{5 3 1} 257$

[38] Frisch M J, Trucks G W, Schlegel H B, Scuseria G E, Robb M A, Cheeseman J R et al 2009 Gaussian 09, revision D.01. Wallington, CT: Gaussian, Inc.

[39] Kresse G and Furthmüller J 1996 Phys. Rev. B 5411169

[40] Kresse G and Hafner J 1993 J. Phys. Rev. B 4813115

[41] Gonze X and Lee C 1997 Phys. Rev. B 5510355 